\title{
Automatic detection and classification of nasopharyngeal carcinoma on PET/CT with support vector machine
}

\author{
Bangxian Wu • Pek-Lan Khong • Tao Chan
}

Received: 19 September 2011 / Accepted: 19 December 2011

(C) The Author(s) 2011. This article is published with open access at Springerlink.com

\begin{abstract}
Purpose Positron emission tomography/computed tomography (PET/CT) has established values for imaging of head and neck cancers, including the nasopharyngeal carcinoma (NPC), utilizing both morphologic and functional information. In this paper, we introduce a computerized system for automatic detection of NPC, targeting both the primary tumor and regional nodal metastasis, on PET/CT.

Methods Candidate lesions were extracted based on the features from both PET and CT images and a priori knowledge of anatomical features and subsequently classified by a support vector machine algorithm. The system was validated with 25 PET/CT examinations from 10 patients suffering from NPC. Lesions manually contoured by experienced radiologists were used as the gold standard.

Results Results showed that the system successfully identified all 53 hypermetabolic lesions larger than $1 \mathrm{~cm}$ in size and excluded normal physiological uptake in brown fat, muscles, bone marrow, brain, and salivary glands.

Conclusion The system combined both imaging features and a priori clinical knowledge for classification between pathological and physiological uptake. Preliminary results showed that the system was highly accurate and promising for adoption in clinical use.
\end{abstract}

Keywords PET/CT - Computer automatic diagnosis . Support vector machine $\cdot$ Nasopharyngeal carcinoma

B. Wu · P.-L. Khong $\cdot$ T. Chan $(\bowtie)$ Department of Diagnostic Radiology,

The University of Hong Kong, Room 406, Block K, Queen Mary Hospital, Pok Fu Lam, Hong Kong

e-mail: taochan@hku.hk

\section{Introduction}

Positron emission tomography/computed tomography (PET/ $\mathrm{CT}$ ) is a hybrid imaging modality comprising both PET (position emission tomography) that images metabolic function in a quantifiable manner and CT (computer tomography) that images body anatomy $[1,2]$. It has become a standard imaging modality for diagnosis and surveillance of various cancers, including head and neck malignancies [3-5]. Compared with PET alone systems, PET/CT is superior in that accurate anatomical localization is possible, which is especially advantageous in the head and neck region where the anatomy is complex and tissues showing physiological uptake are abundant. Still, the variability in metabolism and the large amount of image data make the interpretation of PET/CT a challenging and time-consuming process. Such painstaking processes often need to be repeated when comparison is to be made between examinations for treatment monitoring and response assessment. It is anticipated that an automatic system providing systematic evaluation of PET/CT can help improve the consistency and efficiency.

Over the past two decades, computer-aided diagnosis (CAD) systems have moved from research topics to food and drug administration (FDA)-approved applications used in the clinics [6]. Recently, some computerized systems that aimed to assist the interpretation of PET or PET/CT examinations were developed $[7,8]$. However, to the best of our knowledge, no CAD system that can accurately identify the lesions on PET/CT has been reported yet. Prior attempts have focused on segmentation and classification based on the intensity change on PET images, including the enhancement of segmentation accuracy [9], quantitative evaluation of therapeutic responses [10], and measurements to avoid region leakage [8]. In this project, we have added algorithms that took into account a priori knowledge of the typical 
spread pattern of NPC, which were incorporated into a new classification method using support vector machine (SVM) $[11,12]$. We believe that combination of clinical knowledge about the characteristic distribution of disease with image processing/analysis techniques can improve the accuracy of computerized systems that can become useful for the interpretation of PET/CT examinations. The percentage overlap of malignant lesions identified by the algorithm and those delineated manually by experts were used as indicators of the result.

\section{Materials}

Twenty-five sets of PET/CT examinations from 10 adult patients suffering from NPC were retrospectively collected from the picture archiving and communication system (PACS) of the PET/CT Unit in the University of Hong Kong for the development of the CAD. The examinations were all performed on the same machine (Discovery VCT, GE Healthcare, Piscataway, NJ, USA) using the same standard protocol. Patients fasted for $6 \mathrm{~h}$ prior to the examination. Contrast-enhanced CT scans were obtained (field of view, $40 \mathrm{~cm}$; pixel size, $0.78 \mathrm{~mm}$; spiral CT pitch, 0.984:1; gantry rotation speed, $0.5 \mathrm{~s}$ ). Then $222-370 \mathrm{MBq}$ of fluorodeoxyglucose $\left({ }^{18} \mathrm{~F}-\mathrm{FDG}\right)$ (adjusted according to patient weight) was given intravenously; after an uptake time of $60 \mathrm{~min}$, wholebody PET scan was performed in around $20 \mathrm{~min}$ (6-7 bed positions, $3 \mathrm{~min}$ per bed position). Attenuation correction for PET data using CT images was performed, and images were reconstructed using an ordered-subset expectation maximization iterative algorithm (14 subsets and two iterations). Five patients underwent two PET/CT examinations (one scan before and another after radiotherapy), and five underwent three PET/CT scans (two scans before and one scan after radiotherapy). The images were anonymized before transfer to a separate workstation where the CAD was developed. Institution Review Board (IRB) approval was obtained from our university, and patient consent was waived for this Health Insurance Portability and Accountability Act (HIPAA) compliant study.

All the 25 sets of image slices were randomized and divided into training-validation and testing part with the ratio of $4: 1$. The lesions in each examination were identified by consensus of experienced radiologists as gold standard for subsequent analysis. The lesions were segmented out and labeled as malign or benign manually and the contours further adjusted by the radiologists. Their image features were used to supervise the training progress of the various components from the training dataset identified automatically in the CAD scheme and achieve the final accuracy and overlap rate by comparison with the final estimation result with testing dataset.

\section{Methods}

The CAD program was developed in the MATLAB (The MathWorks, Inc., Natick, MA, USA) programming environment. A schematic diagram of the CAD system is shown in Fig. 1. Our algorithm incorporated the following functions to diagnose NPC automatically.

Locate neck and head positions

The most relevant head and neck region was automatically defined from the series of whole-body CT. In a series of CT slices that included the scan range from head to legs, the pixel numbers of different body compositions were counted in each slice based on the characteristic attenuation of different tissues, namely bone, fat, and soft tissues. This generated a position-intensity curve along the length of the body. Although the exact intensity levels differed between studies, the trend across the body was similar. The index of head and neck could then be identified from this curve by finding the typical pattern along the curve. For example, at the neck, cross-sectional areas of various tissues were all small; therefore, the coincident troughs of all the different tissues located at the level of the neck, which could be consistently demonstrated in all of the 25 cases: the pixel number of all tissues at the neck was around 22,000, while that of the second lowest trough was around 28,000. On the other hand, as facial bones and skull were the main components at the level of the nasopharynx, whereas little soft tissue is present, it was located where the valley point of soft tissues and peak point of bones coexisted. According to the dataset, the pixel number of the soft tissue was about 13,800 , while the second

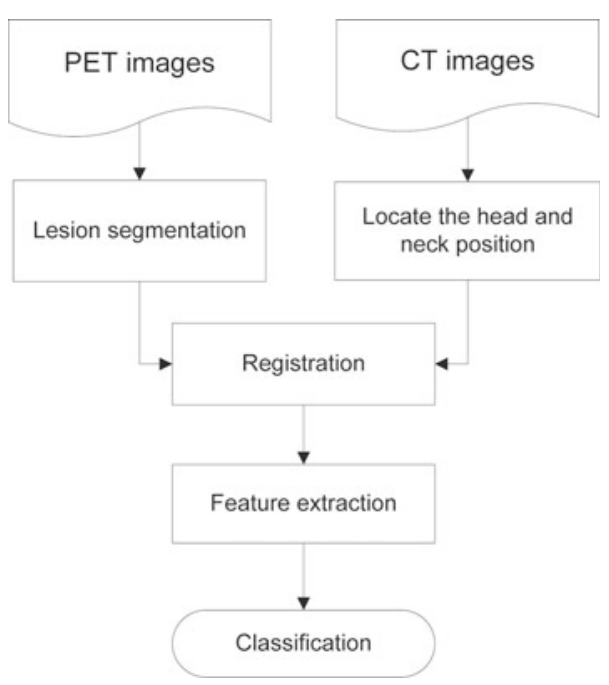

Fig. 1 Schematic diagram shows the workflow of the computer automatic diagnosis system for the classification of malignancy 


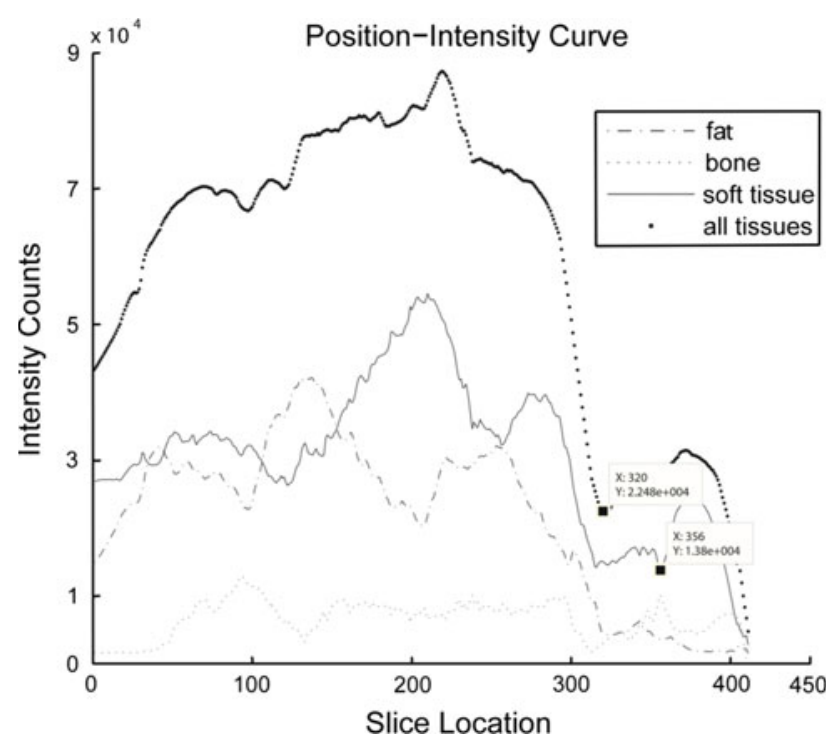

Fig. 2 The position-intensity curve indicates the index of neck and head in a series of whole-body CT images. The counts of fat, bone, and soft tissues are calculated in each slice according to the CT intensity number, and the curve can be plotted along the indices from leg to head. The two valley points in this figure indicate the position indices of neck and head

lowest valley was around 14,500 and the peak was around 52,100. The above processes are illustrated in Fig. 2.

\section{Region growing}

Secondly, the region of interest (ROI) from PET images was segmented using local thresholding and region growing. With the slices selected from neck to head, PET images were used to segment the suspected malignancies. The intensity or pixel value on PET images represents measured radioactivity that can vary according to the use of different imaging techniques. To facilitate the use of the algorithm for images acquired using different techniques, the intensity was converted into standardized uptake value (SUV), using parameters available in the digital imaging and communications in medicine (DICOM) header by the following Eq. (1) [13]:

$$
\mathrm{SUV}=\frac{\text { ROI_counts }}{\text { injected_dose } / \text { body_weight }}
$$

As the SUV values are statistically different between benign and malignant tissues [14], a threshold of calculated SUV could be used to locate seed points within probable malignant lesions. Based on the reported range of SUV in the normal tissues of the head and neck [15-17], cases with recurrent or persistent NPC have significantly higher SUVs (1.6-5.8) than cases with benign lesions $(0.8-1.5)$. Hence, an absolute value of 1.6 was set as the threshold. Such relatively low threshold was used to maintain a high sensitivity of the system. From each PET image, pixels that represented both the regional maximum and carried an SUV value of larger than 1.6 were used as the seed points for subsequent region growing [18].

Forty percent of the value of the seed points was set as the margin for the region growing process based on the training results. Duplicate and connected seed locations were eliminated to reduce the number of loop executions in the program. Strategies adopted for minimizing erroneous inclusion of surrounding tissues in situations included morphological operations and restriction of the greatest difference in area between slices. In addition, regions that had too large an area would be eliminated, which frequently occurred because the intensity of the seed point was not significantly larger than that of other surrounding tissues, suggesting normal rather than pathological tissues.

\section{PET/CT registration}

After the preliminary segmentation on PET images, the next step in the CAD algorithm was to register the suspicious regions on the PET images to its accompanying CT using an algorithm of rigid global registration [19]. The registered CT attenuation data were then utilized to differentiate physiological uptake in some tissues, e.g., brown fat and bone marrow, from the potential tumors, Fig. 3c.

\section{Feature extraction}

After the process of registration, the suspicious regions were identified by regional properties. Image features of these regions were calculated by computer automatically. The shape quantification parameters used in the algorithm included area, relative position, eccentricity, average intensity, symmetry, compactness (the ratio of square of perimeter to area), intensity difference between the regional peak and its circumstance, and second-order textual moments.

Some characteristic combinations of features that suggest physiological uptake in specific tissue types were also considered. The CT unit of bone is usually larger than 180 , and that of fat is less than -30 , hence the average intensity of $\mathrm{CT}$ value in a segmented region was utilized to classify the tumors from physiological marrow uptake in bones and brown fat uptake in fatty tissues. The FDG intensity difference between the regional peak and its surrounding was used as a complementary attribute in other situations, for instance, to differentiate true bone metastasis that tends to be focal from normal bone marrow uptake that tends to be homogeneously distributed.

In addition, anatomical information was used for the classification based on the a priori knowledge of the characteristic distribution and spread pattern of NPC. By definition, the primary tumor of NPC arises from the nasopharynx, and the cancers customarily spread via lymphatic route, with a typical pattern of spread across an expected range of lymph 

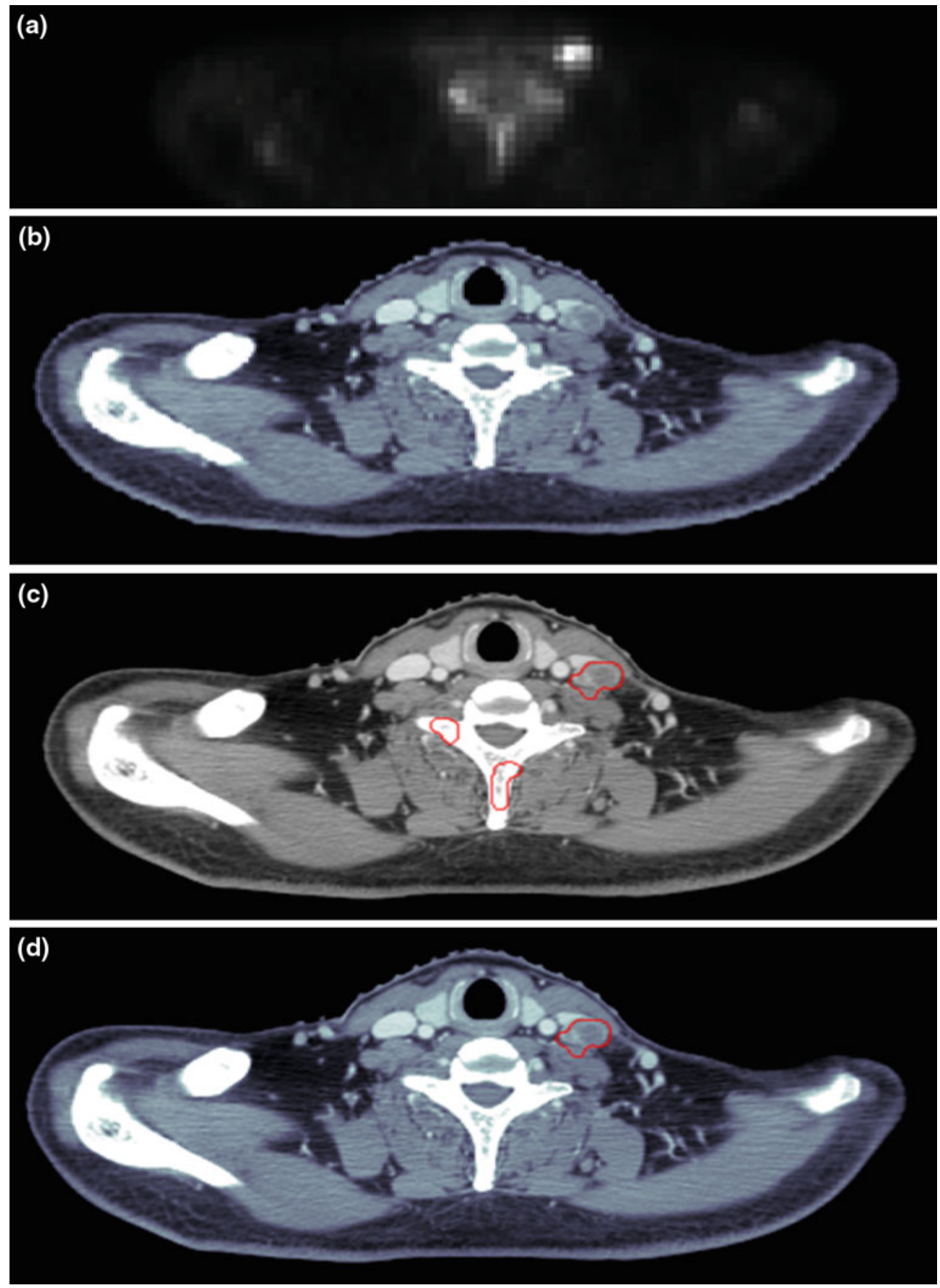

Fig. 3 Images show the main procedures of the scheme. a Original PET image. b Original CT image. $\mathbf{c}$ Registration of the segmented regions to CT image. d Differentiate the malignancy from the bone marrow uptake by support vector machine

nodes in the neck. The likelihood of a segmented candidate to be a part of the primary tumor or its nodal metastasis hence differed according to its anatomical location.

The relative positions $(s, x, y, z)$ could be identified by an anatomical model. The parameter s referred to the different sections in head and neck region, while $(x, y, z)$ are coordinates of the ROI in each specific section $s$. The bones were segmented from the CT images using global thresholding and morphological operations and characteristic features at different anatomical levels were used to define three broad anatomical compartments in head and neck region, namely the neck, oral cavity, and nasal cavity. This rendered information of relative position along the craniocaudal direction to the candidates. Specifically, the jaw bone were the most 
Fig. 4 The figure shows the calculation of the relative position in a specific section. The coordinates $x, y, z$ were all standardized to $0-1$ scale
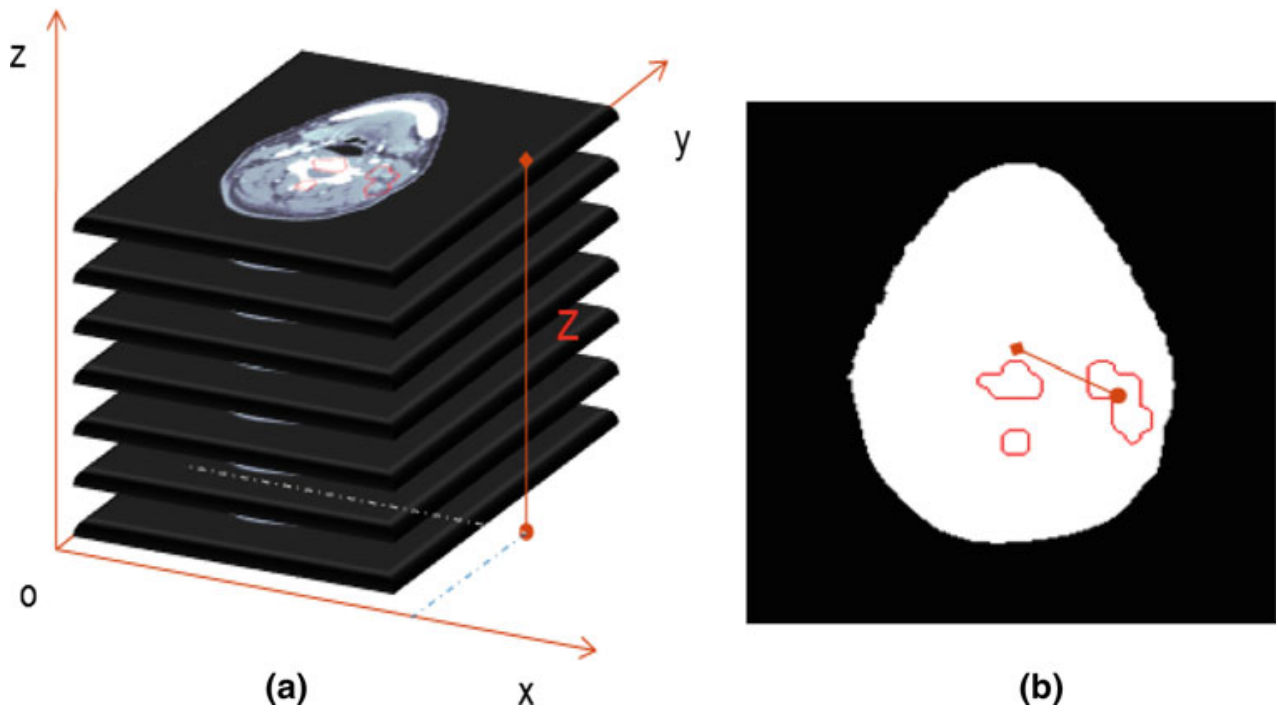

(b) important landmarks, which were recognized as the shape of " $\Lambda$ " at the top part of the CT images; hence, the neck section began with the first slice of the neck and ended with the appearance of the jaw bone, the oral cavity section includes the slices with the existence of the jaw bone, and the nasal section began from the disappearance of the jaw bone. The boundary and the centroid of the head and neck were obtained from the CT images as well. Hence, the parameter s was identified and the candidates were identified into the three above mentioned anatomical compartments using automatically identified fixed bony landmarks in the head and neck. To define a more sophisticated location in each section ' $s$ ', the coordinate $z$ was obtained as the distance from the bottom of the specific section and standardized to $0-1$ scale, and the anatomical locations $(x, y)$ were defined as geometrical coordinates to the centroid in a Cartesian space in the specific anatomical section as illustrated in Fig. 4. Then candidates located at regions where lesions are likely to be found, including the nasal pharynx and different nodal stations, could be identified.

Anatomical information also helped to identify the organs that might show relatively increased FDG uptake, e.g., tonsils, salivary glands, and thyroid. For these, the symmetry of the segmented candidate was taken into consideration as well, e.g., the thyroid gland and salivary glands are normally symmetric about the median plane, and hence symmetrical candidate at such appropriate locations was likely physiological rather than pathological. The bounding box of the candidate was calculated and mapped along the left-right direction about the symmetric axis of the head and neck, and if it overlapped with another ROI, then both candidates were labeled symmetric and vice versa.

All the above mentioned features were tested for developing the classification system after standardization, and only the appropriate ones were selected based on the training results.

\section{Classification}

An SVM [11] incorporating the above mentioned extracted feature vectors has been trained as the classification tool. The SVM classification separated data into training and testing sets, and each instance in the training set contained target value and several attributes. In our scheme, the target value was the different classes of malignancies and physiological uptake or artifacts, and the attributes were the image features extracted [20]. The goal of SVM was to produce a model that predicted the target values of the test data given only the test attributes. The library LIBSVM [21,22] (ref. http://www. csie.ntu.edu.tw/ cjlin/libsvm) has been used for the implementation of the training and prediction procedures of the SVM.

For each instance in the training datasets of our scheme, manually identified lesions by experts were used as gold standard to guide the training process. The attribute vector of SVM included the image features extracted from the ROI as mentioned above. By different combinations of features, physiological uptake could be differentiated from that of the malignancies; for example, uptake of brown fat would coincide in location with fat density tissues on CT, uptake due to muscular contractions is frequently elongated corresponding to the typical shape of muscles, and uptake in salivary glands is usually distributed symmetrically. The exponential function was chosen as the kernel function to map the feature vector into a higher dimensional space as the dimension of the extracted feature vectors was more than four. Compared to the linear kernel, this radial basis function has less 
Table 1 The table shows the fivefold cross-validation accuracy of the image features and their combination

\begin{tabular}{llr}
\hline Combinations of image features & Fivefold cross-validation accuracy \\
\cline { 2 - 3 } & Sensitivity (\%) & False positive (\%) \\
\hline $\begin{array}{l}\text { Position, average intensity, area, eccentricity, symmetry, compactness, } \\
\text { intensity difference, textual moments }\end{array}$ & 90.2 & 11.8 \\
Position, average intensity, area, eccentricity, symmetry, textual moments, & 8.7 \\
$\quad$ intensity difference & 93.4 & 8.5 \\
Position, average intensity, area, eccentricity, symmetry, intensity difference & 93.8 & 4.8 \\
Position, average intensity, area, eccentricity, symmetry & 99.3 & 5.8 \\
Position, average intensity, area, eccentricity & 97.2 & 5.8 \\
Position, average intensity, area, symmetry & 97.4 & 6.5 \\
Position, average intensity, eccentricity, symmetry & 96.7 & 7.1 \\
Position, area, eccentricity, symmetry & 95.1 & 10.1 \\
Average intensity, area, eccentricity, symmetry & 92.3 \\
Area, eccentricity, symmetry & 88.2 & 14.2 \\
\hline
\end{tabular}

The result illustrates that the combination of relative position, average intensity, area, eccentricity, and symmetry has the highest accuracy

hyperparameters that influence the complexity of model selection and can nonlinearly map samples into a higher dimensional space [12]. Other parameters of SVM were selected by the method of fivefold cross-validation, including the appropriate feature vectors. By comparing the accuracy between these different image features, the vector comprising the attributes of area, average intensity, eccentricity, relative position, and symmetry was selected to train the SVM in the final scheme. Other features would comprise a decision tree as a complementary classification to the SVM result.

When the training process was completed, an SVM prediction model was built. A cross-validation was used to verify the efficiency and accuracy compared to the gold standard. All the collected data were used to detect the malignancies. Figure $3 \mathrm{~d}$ shows a classification result of malignancy from the bone artifacts. The final accuracy of the CAD in terms of lesion identification and correct classification was evaluated by comparison with the gold standard. After the classification process, a 3D model of tumors was built from all the 2D classified lesions which was then used for evaluation of the accuracy.

\section{Evaluation test}

The SVM model was identified by fivefold cross-validation test with the training-validation dataset. The image features and SVM parameters producing the highest average accuracy of the fivefolds were selected and used in our scheme. Then, the 2D classification result on the testing dataset was compared to the gold standard.

In terms of the 3D classification, lesions were identified as discontinuous volumes in the 2D PET/CT ROIs, and there were 53 lesions in all the 10 patients, ranging from 6 in case $1-0$ (after radiotherapy) in case 10 . For the evaluation, we defined a positive identification if a lesion volume produced by the algorithm overlapped at least $80 \%$ of the lesion volume drawn by radiologists.

\section{Results}

2D evaluation test result

By a fivefold leave-one-out cross-validation test with randomization of all the suspicious regions segmented from the 20 sets of imaging data from eight patients, the average sensitivity and false-positive rate of the fivefolds are listed in Table 1 . The image feature combination of relative position, average intensity, area, eccentricity, and symmetry has the highest accuracy, and the relative position is significantly the most important feature. The confusion matrix of the SVM cross-evaluation with the image features mentioned above is listed in Table 2. The classification result with the testing data is listed in Table 3. The final sensitivity of the CAD scheme with the testing data was $95.1 \%$ and the false-positive rate was $7.0 \%$. Accuracy of the SVM was $93.3 \%$, while the original segmentation result accuracy without SVM was only $22.3 \%$.

Table 2 The table shows the confusion matrix of cross-validation result of the classification of the 20 sets of training data

\begin{tabular}{lrrr}
\hline Count & \multicolumn{2}{l}{ Predicted } & Total \\
\cline { 2 - 3 } & Malignancies & Artifacts & \\
\hline Gold Standard & & & \\
Malignancies & 159 & 7 & 166 \\
Artifacts & 8 & 1,149 & 1,157 \\
Total & 167 & 1,156 & 1,323 \\
\hline
\end{tabular}


Table 3 The table shows the confusion matrix of the classification result of the five sets of testing data

\begin{tabular}{llcr}
\hline Count & Predicted & Total \\
\cline { 2 - 3 } & Malignancies & Artifacts & \\
\hline Gold Standard & & & \\
Malignancies & 58 & 3 & 61 \\
Artifacts & 19 & 250 & 269 \\
Total & 77 & 253 & 330 \\
\hline
\end{tabular}

\section{D classification result}

Based on the comparison with manual segmentation by an experienced radiologist, the system identified all the 53 lesions in all 25 cases on an $80 \%$ coverage level. It also successfully excluded normal physiological uptake in brown fat, muscles, bone marrow, brain, and salivary glands via the SVM classification procedure in all cases, including training and testing datasets.

But in the total 25 cases of the dataset, five asymmetrical and unusually hot tonsils and larynx were mistakenly reported as lesions. The false-positive rate was $8.6 \%$ based on the 3D model result.

\section{Discussion}

Magnetic resonance imaging (MRI) is imaging modality of choice evaluation of NPC due to its high spatial resolution, especially for the assessment of locoregional invasion and retropharyngeal nodal metastasis in NPC patients. However, previous studies showed that in interpreting MRI images of NPC, lymph nodes of borderline size, without nodal necrosis or extracapsular spread, always pose a diagnostic challenge to the radiologists $[23,24]$. PET/CT is more accurate than MRI for determining cervical nodal metastasis and a better reference for the neck status [25]. In addition, PET/CT is highly sensitive for the detection of distant metastasis and can play an important role in the evaluation of NPC.

A small number of reports on research of computeraided detection and decision support for whole-body PET or PET/CT imaging are available in the literature. There were computer-aided detection systems of FDG avid lesions on PET [7] and PET/CT [26]. These studies located regions showing high FDG uptake, based on the intensity levels above background and/or comparison between both sides. These appeared to be early developments with little supportive evidence of feasibility for clinical use. Recently, a system that automatically evaluated deviation of FDG uptake from normal distribution obtained from screening population was reported [27]. This work has hinted but not addressed the problem of classification of lesions into genuine ones or mimickers and hence may not be efficiently used for decision support in clinical setting. A system that tracked lesions showing FDG uptake on serial following and measures SUV of the same lesion over time has been reported [8]. This system registered the serial examinations against one another and locates lesions by looking for regions of high SUV. The limitation is that this system still required user input to define the index lesion in the first place, meaning that it is not useful for initial diagnosis.

Compared with the previous works, the main contribution of our scheme was the application of the clinical a priori knowledge to differentiate between physiologic and pathologic FDG accumulation via SVM automatic classification. As cancers frequently spread by direct invasion or via lymphatic route, the likelihood of malignancies varies for different anatomical locations; hence, the relative position is a most important feature for identifying tumors. Other features, including the CT intensity, symmetry, and eccentricity, can all become useful for the identification of specific mimics or artifacts.

Figure 5 shows an example of salivary and thyroid glands. In addition to their characteristic anatomical locations, these normal tissues are frequently symmetric, especially the thyroid glands and salivary glands, while the tumors are usually asymmetric. Hence, the scheme classified malignancies from these artifacts successfully with the attributes of symmetry and locations, which are used by the interpreting physicians often. Figure 6 shows an example of brain and muscle. Prominent FDG uptakes within the muscles are usually linear, while the lesions are often as circular as the lymph nodes. Hence, the physiological uptake can be recognized by our scheme, with the attribute of eccentricity in SVM. Figure $7 \mathrm{a}-\mathrm{c}$ shows an example of true-positive and true-negative case of classification.

\section{Robustness of the algorithm}

One major challenge of a useful CAD scheme is to achieve high robustness despite patient variability. A large database of normal FDG distribution in healthy subjects can improve the robustness of the algorithm by identifying differences between diseased and normal individuals. Without the benefit of having such database, the current algorithm that only segments ROIs having significantly high uptake would be selected as candidate. Also, since all the image features of the ROI were standardized to $0-1$ scale before classification to eliminate the differences between patients, including area and relative position, some individual variations related to body habitus are avoided.

The algorithm can be readily applicable for images produced from different manufacturers, since it worked on standard DICOM format with functions that normalized signal intensities. However, as the segmentation process relied on 
Fig. 5 Examples of the salivary and thyroid glands. a Original PET image. b Salivary glands and malignancy are suspected as lesions after registration. c The malignancy is classified successfully from the salivary glands. d Original PET image with thyroid uptake. e Thyroid glands and malignancy are suspected as lesions after registration. f Successful classification of malignancy from the thyroid glands via support vector machine
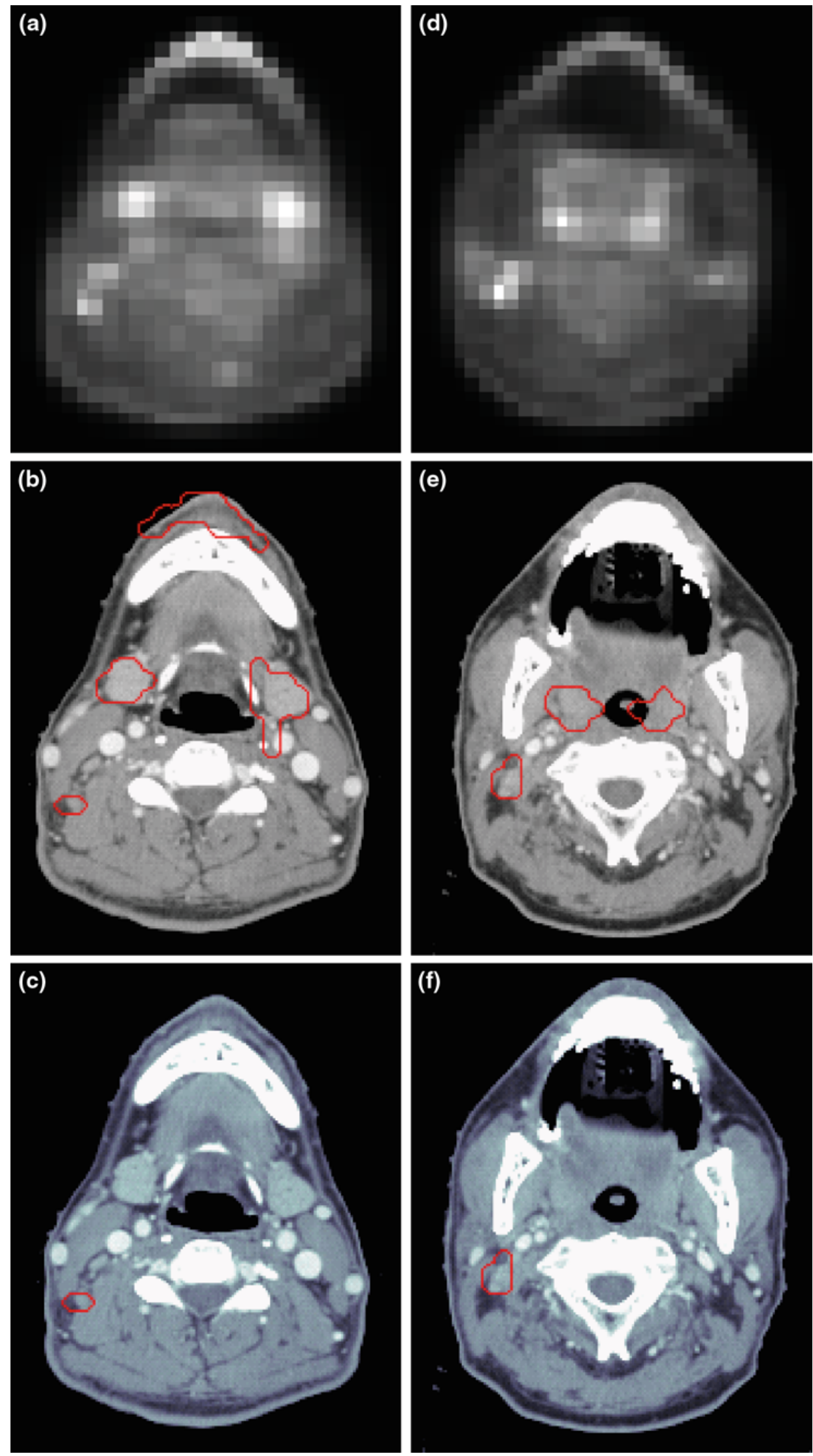
Fig. 6 Examples of brain and muscle. a Original PET image. b Brain and malignancy are suspected as lesions after registration. c The malignancy is classified successfully from the brain. d Original PET image with muscle uptake. e Muscle and malignancy are suspected as lesions after registration. $\mathbf{f}$ Successful classification of malignancy from the muscle via support vector machine
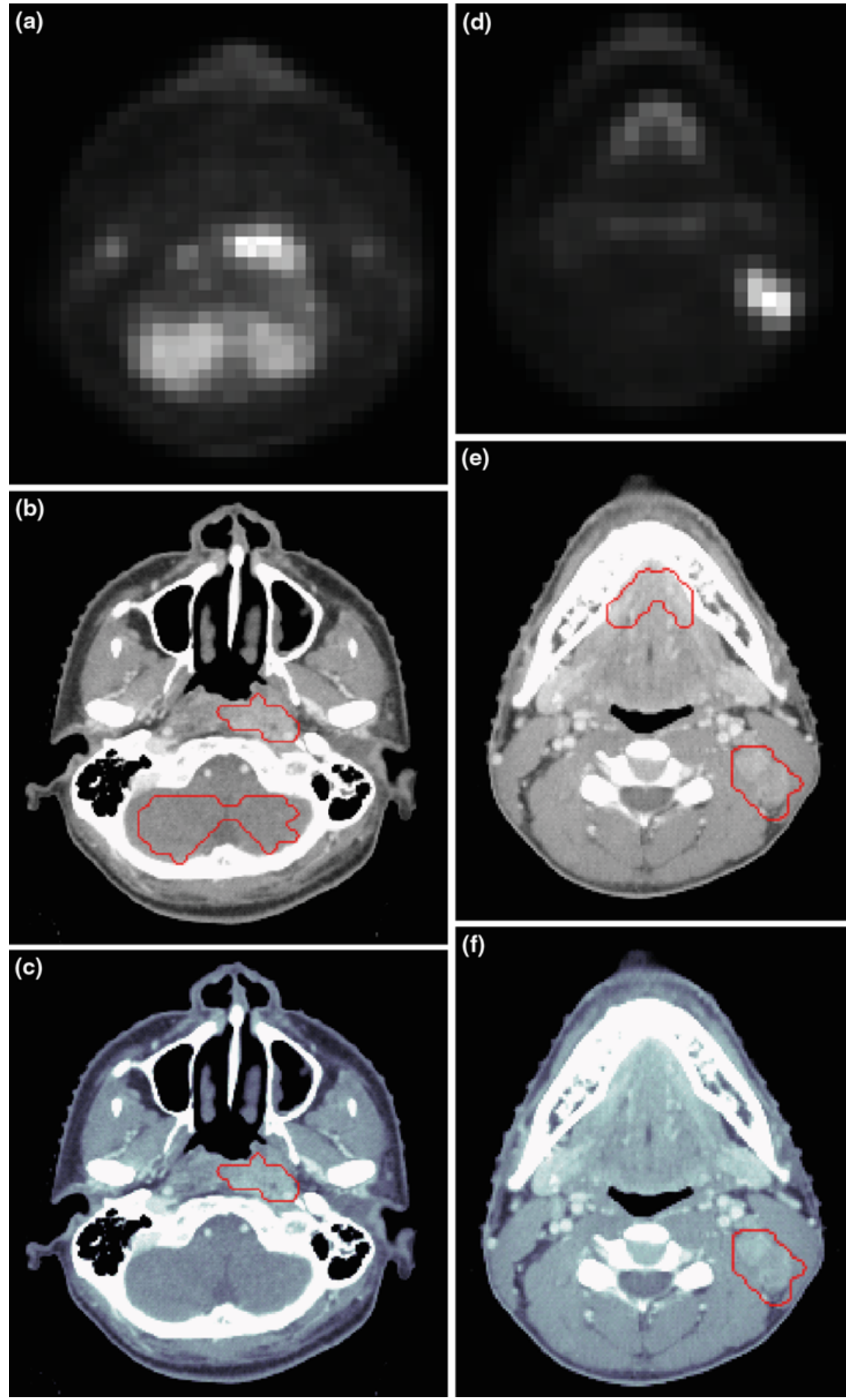

the uptake threshold, scanning techniques or patient factors that might alter uptake would affect the final results. For instance, the intrinsic sensitivity of the machines or the use of different scanning protocol could affect the measured radioactivity significantly, which would require adjustment of the absolute threshold values or conversion of such threshold to a relative function of scanning parameters. Also, scanning patients at different time points after treatment may 

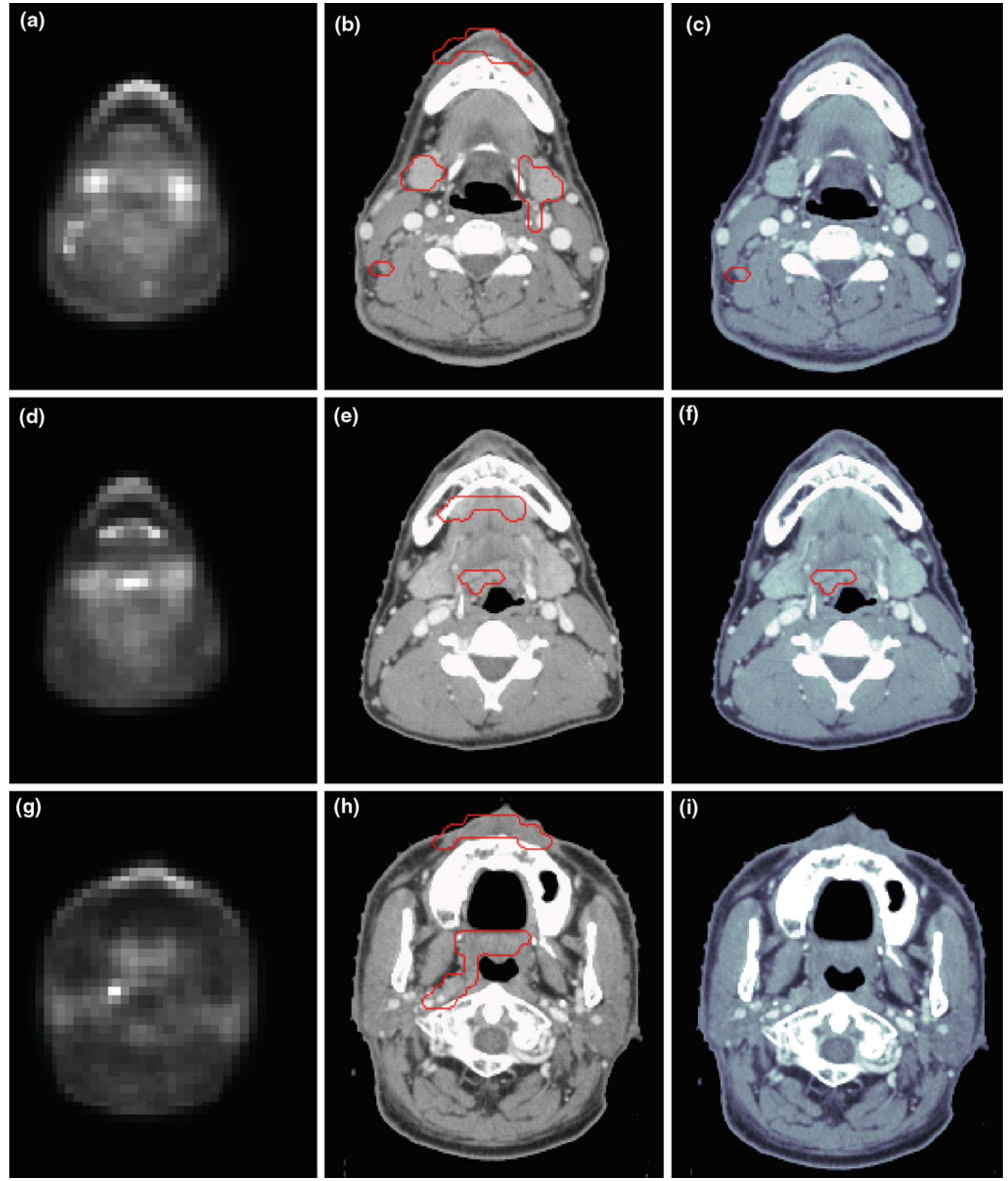

Fig. 7 Examples of true and false classification results. a Original PET images. b Registration results. Tumors, muscle artifacts, and salivary glands are segmented as candidates. $\mathbf{c}$ Both the malignancy and salivary glands are classified successfully. d Original PET images. e Registration result with muscle and larynx artifact as candidates. $\mathbf{f}$ Larynx is recog- nized as a false-positive case. $\mathbf{g}$ Original PET images. $\mathbf{h}$ Registration result. The malignancy and soft palate are misrecognized as one tissue. i A false-negative case that the malignancy could not be classified from the soft palate 
be variably affected by post-treatment changes, e.g., FDG uptake due to post-radiotherapy inflammatory changes that tend to wear off with time.

There were circumstances where intrinsic limitations of PET/CT lead to erroneous classification by the CAD [28]. For example, there could be false negatives when the uptake malignancy was unexpectedly low due to the small size or necrosis, leading to reduced number of metabolically active cells per voxel; there could be false negatives or false positives where there were significant body movements between the PET and CT scan leading to misregistration. Misclassification could occur when the listed image attributes could not differentiate the artifacts from malignancies. A case of false-positive misclassification is shown in Fig. 7d-f, as the attributes of the larynx artifact in this slice were quite similar with the malignancies. Figure $7 \mathrm{~g}-\mathrm{i}$ shows an example of false-negative misclassification. The malignancy could not be identified based on the image features of the combined ROI.

Collection of a large sample of clinical examinations will better determine the applicability and accuracy of the system. Also, comparison between clinicians' performance with and without use of this system can resolve issues such as significance of missed lesions on the CAD and whether or not the use of the CAD complements expert human readers.

\section{Time complexity}

This CAD scheme consisted of the following major components: region growing segmentation, rigid registration, image feature extraction, and SVM classification. For a case of $m$ slices with $n$ pixels in each slice, the whole complexity became $m$ times of that in each slice.

In automatic region growing method, seeds were selected by threshold with a complexity $O(n)$. Checking the region margins and growing could be done in constant time. As each unclassified pixel was inserted into the sorted class-labeled list once, the time complexity of the growing process was $O(n \log n)$. In addition, it took $O(n p)$ to calculate the difference between the regions and merge the similar ones, where $p$ was the number of regions. Hence, the time complexity of each slice segmentation was $O(n p+n \log n)$ [29].

In global rigid registration, the margin of the neck or head was obtained by threshold and image dilation, and hence the time complexity was $O(n)$. For the image feature extraction of each ROI, it took constant time to calculate all the features including area, centroid point, and eccentricity in MATLAB toolbox since both the number of ROIs and the pixel number in each ROI were much smaller than $n$.

In terms of the SVM classification, the standard SVM training has $O\left(q^{3}\right)$ time complexity, where $q$ is the training set size [30,31]. In our scheme, the training set size is the number of ROIs that ranges from zero to around ten each slice. Hence, $\mathrm{q}$ is $O(m)$ and the training time complexity is about $O\left(\mathrm{~m}^{3}\right)$. The prediction process of the LibSVM is $O(m * n)$ [21] with trained support vectors.

Hence, for a case of $\mathrm{m}$ slices with $\mathrm{n}$ pixels in each slice, the time complexity of our scheme to classify the nasopharyngeal carcinoma was $O(m * n \log n)$ with a $O\left(m^{3}\right)$ time to train the SVM in advance.

\section{Conclusion}

An automatic algorithm for identifying NPC on PET/CT examination was developed. It combined both imaging features and a priori clinical knowledge for classification between pathological and physiological uptake. Preliminary results showed that the system was highly accurate and promising for adoption in clinical use.

Conflict of interest None.

Open Access This article is distributed under the terms of the Creative Commons Attribution Noncommercial License which permits any noncommercial use, distribution, and reproduction in any medium, provided the original author(s) and source are credited.

\section{References}

1. Kapoor V, McCook BM, Torok FS (2004) An introduction to PETCT imaging. Radiographics 24(2):523-543

2. Townsend DW et al (2004) PET/CT today and tomorrow. J Nucl Med 45(Suppl 1):4S-14S

3. Weber WA, Figlin R (2007) Monitoring cancer treatment with PET/CT: does it make a difference? J Nucl Med 48(Suppl 1):36S$44 \mathrm{~S}$

4. Lee SW et al (2008) Prediction of prognosis using standardized uptake value of 2-[(18)F] fluoro-2-deoxy-d-glucose positron emission tomography for nasopharyngeal carcinomas. Radiother Oncol 87(2):211-216

5. Schinagl DA et al (2011) Can FDG PET predict radiation treatment outcome in head and neck cancer? Results of a prospective study. Eur J Nucl Med Mol Imaging 38(8):1449-1458

6. Doi K (2005) Current status and future potential of computer-aided diagnosis in medical imaging. Br J Radiol 78(Spec No 1):S3-S19

7. Tozaki YT et al (2003) Computer assisted diagnosis method of whole body cancer using FDG-pet images. In: Proceedings of international conference on image processing, vol 2, pp 1085-1088

8. Opfer R et al (2008) Automatic lesion tracking for a PET/CT based computer aided cancer therapy monitoring system. In: Giger ML, Karssemeijer N (eds) Medical imaging 2008: computer-aided diagnosis. Proceedings of the SPIE, vol 6915, pp 691513.1-691513.10

9. Sharif MS et al (2010) Artificial neural network-based system for PET volume segmentation. Int J Biomed Imaging 2010

10. Gao X et al (2010) Computer-assisted quantitative evaluation of therapeutic responses for lymphoma using serial PET/CT imaging. Acad Radiol 17(4):479-488

11. Cortes C, Vapnik V (1995) Support-vector networks. Mach Learn 20(3):273-297

12. Suykens JAK, Vandewalle J (1999) Least squares support vector machine classifiers. Neural Process Lett 9(3):293-300 
13. Thie JA (2004) Understanding the standardized uptake value, its methods, and implications for usage. J Nucl Med 45(9):14311434

14. Aoki J et al (2001) FDG PET of primary benign and malignant bone tumors: standardized uptake value in 52 lesions. Radiology 219(3):774-777

15. Kao CH et al (1998) Detection of recurrent or persistent nasopharyngeal carcinomas after radiotherapy with 18-fluoro-2deoxyglucose positron emission tomography and comparison with computed tomography. J Clin Oncol 16(11):3550-3555

16. Yen TC et al (2005) Are dual-phase 18F-FDG PET scans necessary in nasopharyngeal carcinoma to assess the primary tumour and loco-regional nodes? Eur J Nucl Med Mol Imaging 32(5):541-548

17. Allal AS et al (2002) Standardized uptake value of 2-[F-18] fluoro-2-deoxy-D-glucose in predicting outcome in head and neck carcinomas treated by radiotherapy with or without chemotherapy. J Clin Oncol 20(5):1398-1404

18. Pham DL, Xu CY, Prince JL (2000) Current methods in medical image segmentation. Annu Rev Biomed Eng 2:315-337

19. Hill DLG et al (2001) Medical image registration. Phys Med Biol 46(3):R1-R45

20. Furey TS et al (2000) Support vector machine classification and validation of cancer tissue samples using microarray expression data. Bioinformatics 16(10):906-914

21. Chang C, Lin CJ (2001) LIBSVM: a library for support vector machines. http://www.csie.ntu.edu.tw/ cjlin/papers/libsvm.pdf

22. Hsu C, Chang CC, Lin CJ (2003) A practical guide to support vector classification. http://www.csie.ntu.edu.tw/ cjlin/papers/guide/ guide.pdf
23. $\mathrm{Ng} \mathrm{SH}$ et al (2004) Nodal metastases of nasopharyngeal carcinoma: patterns of disease on MRI and FDG PET. Eur J Nucl Med Mol Imaging 31(8):1073-1080

24. Ng SH et al (2009) Staging of untreated nasopharyngeal carcinoma with PET/CT: comparison with conventional imaging workup. Eur J Nucl Med Mol Imaging 36(1):12-22

25. King AD et al (2008) The impact of 18 F-FDG PET/CT on assessment of nasopharyngeal carcinoma at diagnosis. Br J Radiol 81(964):291-298

26. Aung $\mathrm{W}$ et al (2005) In-vivo PET imaging of inducible D2R reporter transgene expression using [11C]FLB 457 as reporter probe in living rats. Nucl Med Commun 26(3):259-268

27. Hara $\mathrm{T}$ et al (2008) Automated scoring system of standard uptake value for torso FDG-PET images. Proc SPIE 6915:691534691534-4

28. Griffeth LK (2005) Use of PET/CT scanning in cancer patients: technical and practical considerations. Proc (Bayl Univ Med Cent) 18(4):321-330

29. Shih FY, Cheng SX (2005) Automatic seeded region growing for color image segmentation. Image Vis Comput 23(10):877-886

30. Loosli G, Canu S (2007) Comments on the "core vector machines: fast SVM training on very large data sets". J Mach Learn Res 8:291-301

31. Tsang IW, Kwok JT, Cheung PM (2005) Core vector machines: fast SVM training on very large data sets. J Mach Learn Res 6:363392 